This article was downloaded by: [Universita degli Studi di Torino]

On: 05 J une 2013, At: 05:22

Publisher: Routledge

Informa Ltd Registered in England and Wales Registered Number: 1072954 Registered office: Mortimer House, 37-41 Mortimer Street, London W1T 3J H, UK

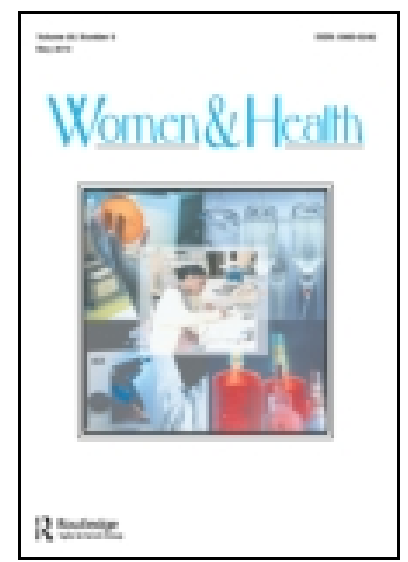

\author{
Women \& Health \\ Publication details, including instructions for authors and \\ subscription information: \\ http:// www. tandfonline.com/loi/ wwah20
}

\title{
Different Types of Physical Activity on Inflammatory Biomarkers in Women With or Without Metabolic Disorders: A Systematic Review
}

\author{
Fabiano Henrique Rodrigues Soares MD ${ }^{a} \&$ Maria Bernardete \\ Cordeiro de Sousa PhD ${ }^{b}$ \\ a Health Science Center, Federal University of Rio Grande do Norte, \\ Natal, Rio Grande do Norte, Brazil \\ ${ }^{b}$ Physiology Department, Federal University of Rio Grande do \\ Norte, Natal, Rio Grande do Norte, Brazil \\ Accepted author version posted online: 13 Mar 2013. Published \\ online: 24 May 2013.
}

To cite this article: Fabiano Henrique Rodrigues Soares MD \& Maria Bernardete Cordeiro de Sousa PhD (2013): Different Types of Physical Activity on Inflammatory Biomarkers in Women With or Without Metabolic Disorders: A Systematic Review, Women \& Health, 53:3, 298-316

To link to this article: http:// dx.doi.org/ 10.1080/03630242.2013.782940

\section{PLEASE SCROLL DOWN FOR ARTICLE}

Full terms and conditions of use: http://www.tandfonline.com/page/terms-and-conditions

This article may be used for research, teaching, and private study purposes. Any substantial or systematic reproduction, redistribution, reselling, loan, sub-licensing, systematic supply, or distribution in any form to anyone is expressly forbidden.

The publisher does not give any warranty express or implied or make any representation that the contents will be complete or accurate or up to date. The accuracy of any instructions, formulae, and drug doses should be independently verified with primary sources. The publisher shall not be liable for any loss, actions, claims, proceedings, demand, or costs or damages whatsoever or howsoever caused arising directly or indirectly in connection with or arising out of the use of this material. 


\title{
Different Types of Physical Activity on Inflammatory Biomarkers in Women With or Without Metabolic Disorders: A Systematic Review
}

\author{
FABIANO HENRIQUE RODRIGUES SOARES, MD \\ Health Science Center, Federal University of Rio Grande do Norte, \\ Natal, Rio Grande do Norte, Brazil \\ MARIA BERNARDETE CORDEIRO DE SOUSA, PhD \\ Physiology Department, Federal University of Rio Grande do Norte, \\ Natal, Rio Grande do Norte, Brazil
}

\begin{abstract}
Overwhelming data support the relationship between physical inactivity and inflammatory processes underlying metabolic disorders. The aim of this review was to assess critically the body of evidence for the relationships between exercise protocols and levels of inflammatory biomarkers in women. Systematic literature searches using PubMed Medline, Cochrane Central Register of Controlled Trials, Web of Science, LILACS, and SciELO of publications from 1993 to January 2012 were conducted using the following keywords: inflammation, cytokine, exercise, physical training, aerobic training, cardiovascular training, strength training, resistance training, interval training, cardiac rehabilitation, and therapeutic lifestyle modification/management. Three studies reported no significant changes in inflammatory status, one documented an increase in inflammatory biomarkers, and 12 articles reported decreased inflammatory biomarkers associated with exercise. Secondary lifestyle characteristics, such as strenuous physical labor or smoking, were correlated with levels of inflammatory biomarkers. Integrative interventions, including diet, moderate aerobic (60\% to $80 \%$ of maximum heart
\end{abstract}

Received August 9, 2012; revised February 25, 2013; accepted March 2, 2013.

The authors declare no conflict of interest.

Address correspondence to Fabiano Henrique Rodrigues Soares, MD, Rua Francisco Pignataro, 1942 ap. 201 Capim Macio, Natal, RN 59082-070, Brazil. E-mail: fsfitness@ hotmail.com 
rate, or $50 \%$ to $60 \%$ of $V_{2} O_{2 x a x}$ ) and circuit resistance training ( 8 to 10 exercises, 8 to 12 repetitions), bealth education, and counseling, used together, appeared to be effective strategies to improve inflammatory biomarkers in women.

KEYWORDS cardiovascular disease, physical activity, risk factors

\section{INTRODUCTION}

Physical activity has been associated with protection against development of several chronic disorders, such as metabolic syndrome (MS) (Troseid et al., 2009). According to Booth et al. (2002), through evolution, metabolic pathways have developed in which human genes were selected to allow aerobic metabolism and to orchestrate the complex integration needed for physical activity, making it possible to survive during food acquisition, defense, and reproduction (Booth, Chakravarthy, \& Spangenburg, 2002). Regular exercise may offer protection against a number of chronic diseases (Smith et al., 2008; Silva et al., 2008; Skilton et al., 2008).

In addition to being an energy depot, fat mass is also an endocrine organ (Garruti et al., 2008) and has a role in producing inflammatory biomarkers that could be involved in the pathogenetic molecular mechanism that connects augmented fat mass to obesity-associated comorbidities (Lacasa et al., 2007; Lazar, 2005; Bengmark, 2006). The decrease in adipose tissue mass through weight reduction has been associated with a reduction in levels of tumor necrosis factor (TNF- $\alpha$ ), interleukin 6 (Il-6), plasminogen activator inhibitor (PAI-1), C-reactive protein (CRP), and other inflammatory biomarker levels associated with improvements in insulin sensitivity and endothelial function (Aldhahi \& Hamdy, 2003). Given the marked heterogeneity of exercise protocols and methods, choosing the most effective exercise program for reducing the consequences of increases in inflammatory markers and increasing the protective effects of exercise depends on a variety of variables. Therefore, the aim of this review was to identify relevant studies using a systematic search-and-review procedure to provide a critical review of the relations of different types of acute and chronic physical activity to inflammatory biomarkers in females.

\section{METHODS}

\section{Search Strategy}

A systematic search was conducted to identify potentially relevant articles published from 1993 to January 2012, using five electronic databases (PubMed Medline, Cochrane Central Register of Controlled Trials, Web of Science, LILACS, and SciELO). The search strategy included a mix 
of $\mathrm{MeSH}$ and free-text terms in English, Portuguese, and Spanish for the following keywords: inflammation, cytokine, exercise, physical training, aerobic training, cardiovascular training, strength training, resistance training, interval training, cardiac rehabilitation, and therapeutic lifestyle modification/management. Pooling of effect sizes across studies in a metaanalysis was inappropriate at this time, given the heterogeneity of the physical activity protocols and the paucity of robust randomized controlled trials (RCTs).

\section{Inclusions}

Randomized, controlled or uncontrolled experimental trials published in peer-reviewed journals were included if they met the following criteria: analyzed physical activity or therapeutic lifestyle management of metabolic disorders, with primary or secondary outcome measures of pro- or antiinflammatory biomarkers in healthy or unhealthy females. Observational studies were not included.

\section{Exclusions}

Animal studies were excluded, as were studies involving pharmacologic interventions. Studies that did not evaluate the desired outcome measures or included children, males, or adolescents were also excluded, in addition to those with Physiotherapy Evidence Database (PEDro) scores lower than three (Verhagen et al., 1998; De Morton, 2009). The PEDro scale, a measure of the methodological quality of clinical trials, is based on the Delphi List developed by Verhagen et al. (1998). Additional quality variables considered for exclusion were lack of descriptions about exercise training supervision, lifestyle modification protocols, or acute bout sessions, as well as inadequate reporting of exercise modality and methods used in inflammatory biomarkers assays.

\section{Categorization of Studies}

Eligible articles were classified into one of five categories of exercise intervention: therapeutic lifestyle management (TLM), aerobic training (AT), resistance training (RT), cardiac rehabilitation (CR), and acute aerobic exercise (AAE).

\section{Data Synthesis and Analysis}

Researchers synthesized data related to inflammatory biomarkers in women, type of acute physical activity, type of physical training, or lifestyle 
modification protocols. Additional quality assessments included proper randomization, adequate sample sizes, methods of sample selection, sources of potential biases, standardization of data collection and instruments, appropriate length of follow-up, and adequate statistical analysis as summarized in the CONSORT Statement (Schulz et al., 2010), without providing further scoring classification. Primary outcome measures following physical activity or therapeutic lifestyle modification were post-intervention changes in inflammatory biomarkers. The relationships between changes in these outcomes measures and different physical activity protocols were also analyzed. Researchers requested missing data from one author but received no response. Data values were converted to percentages to standardize the analysis.

\section{RESULTS}

\section{Relevant Studies}

The search generated 83 relevant citations (Figure 1). Researchers accepted 16 studies according to the inclusion/exclusion criteria and excluded 54 with male participants, three with adolescents, two for using drug intervention, three for being literature reviews, and five due to low PEDro scale scores. Experimental studies consisted of five articles on TLM, six on AT, three on $\mathrm{RT}$, one on AAE, and one on $\mathrm{CR}$ in relation to inflammatory biomarkers. Nine studies were uncontrolled trials (UC), five were RCTs, and two were

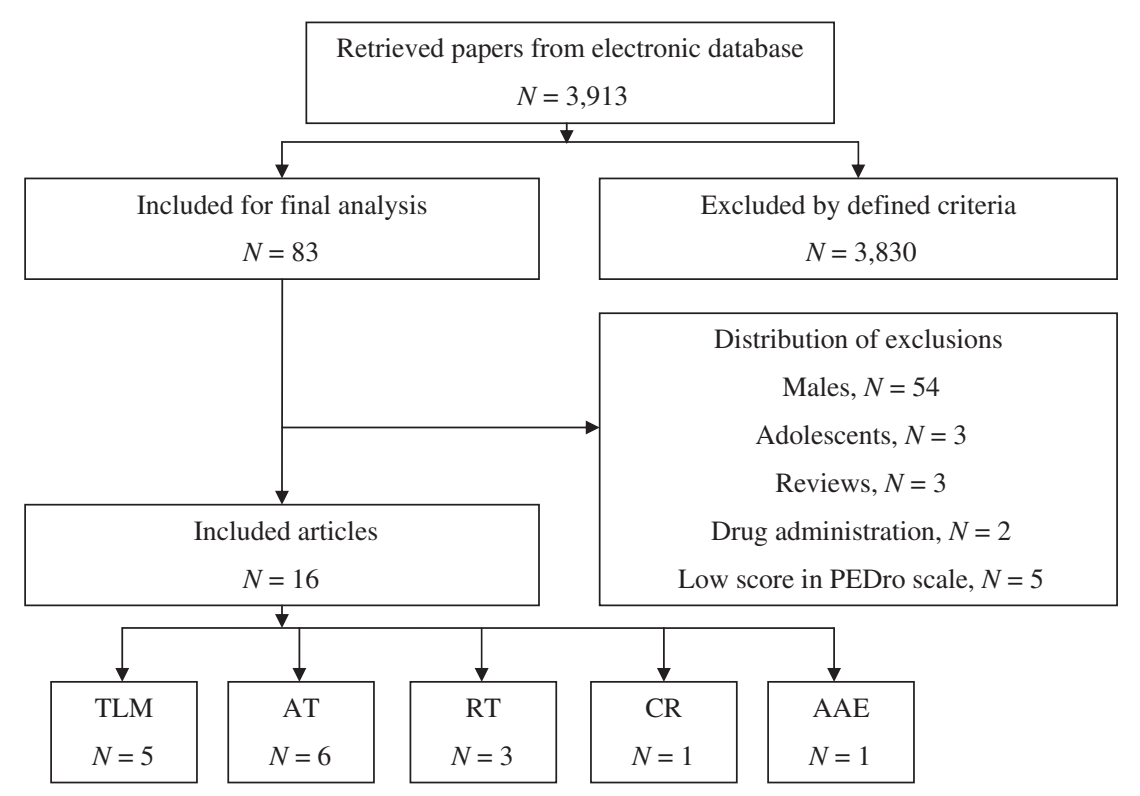

FIGURE 1 Summary of search results. 
non-randomized controlled clinical trials (NRCTs). Three studies reported no change in inflammatory status, one found an increase in inflammatory biomarkers associated with exercise, and 12 articles reported decreases in inflammatory markers (Table 1).

TLM protocols often included exercise, diet, and psychosocial interventions representing integrative/interdisciplinary approaches. TLM is advantageous in lifestyle management as it covers a broader range of life aspects as well as CR programs. Use of an RT intervention was associated with a significant decrease in adiponectin levels, averaging about 3.7\%. No significant changes occurred in other biomarkers with an RT intervention. CR appeared to be the most effective protocol in decreasing inflammatory biomarkers with a mean reduction over all markers (TNF- $\alpha$, high-sensitivity CRP [hs-CRP], Il-6, and intracellular adhesion molecule 1 [ICAM-1]) of 45.2\%, with hs-CRP and TNF- $\alpha$ declining by $40 \%$ and Il- 6 by $55 \%$. Studies of AT reported the following mean decreases in markers after interventions: leptin $=38.7 \%$, hs$\mathrm{CRP}=30.0 \%, \mathrm{CRP}=34.9 \%$, Il-6 $=23.0 \%$, and interferon-gamma $($ IFN- $\gamma)=$ $27.6 \%$. One study reported statistically significant differences in inflammatory biomarkers before and after AAE which were associated with an increase in IFN- $\gamma$ of $92.5 \%$ and in Il-6 of $43.5 \%$, with a total mean increase of $68 \%$ over all inflammatory markers.

\section{CR}

Only one study involved a CR program (Beckie, Beckstead, \& Groer, 2010) and investigated a 12-week, behaviorally-enhanced CR exclusively for women compared to traditional $\mathrm{CR}$, several components of the MS, and inflammatory biomarkers in women with coronary heart disease (CHD). The traditional CR program was nationally certified by the American Association of Cardiovascular and Pulmonary Rehabilitation (AACVPR) and included an exercise protocol of AT three days per week (60\%-85\% of maximal heart rate) plus eight hours of education classes, focusing on CHD risk factor modification. The gender-tailored protocol was identical to the traditional CR program, except that the participants exercised only with women, and female research nurses applied motivational interviewing and screening for motivational readiness to modify physical and dietary habits and addressed stress reduction, providing an individualized report with feedback on each behavior that must be changed. In addition, participants underwent a psychosocial intervention to influence behavioral change with psychoeducational classes focused on gender-based practice guidelines, relaxation exercises, and social support. Data collected before and after the intervention showed that the total sample evidenced significant reductions in inflammatory biomarkers. However, the women-specific CR program promoted greater improvements in inflammatory biomarkers than the traditional CR program. The gendertailored intervention was also superior in reducing some components of MS 


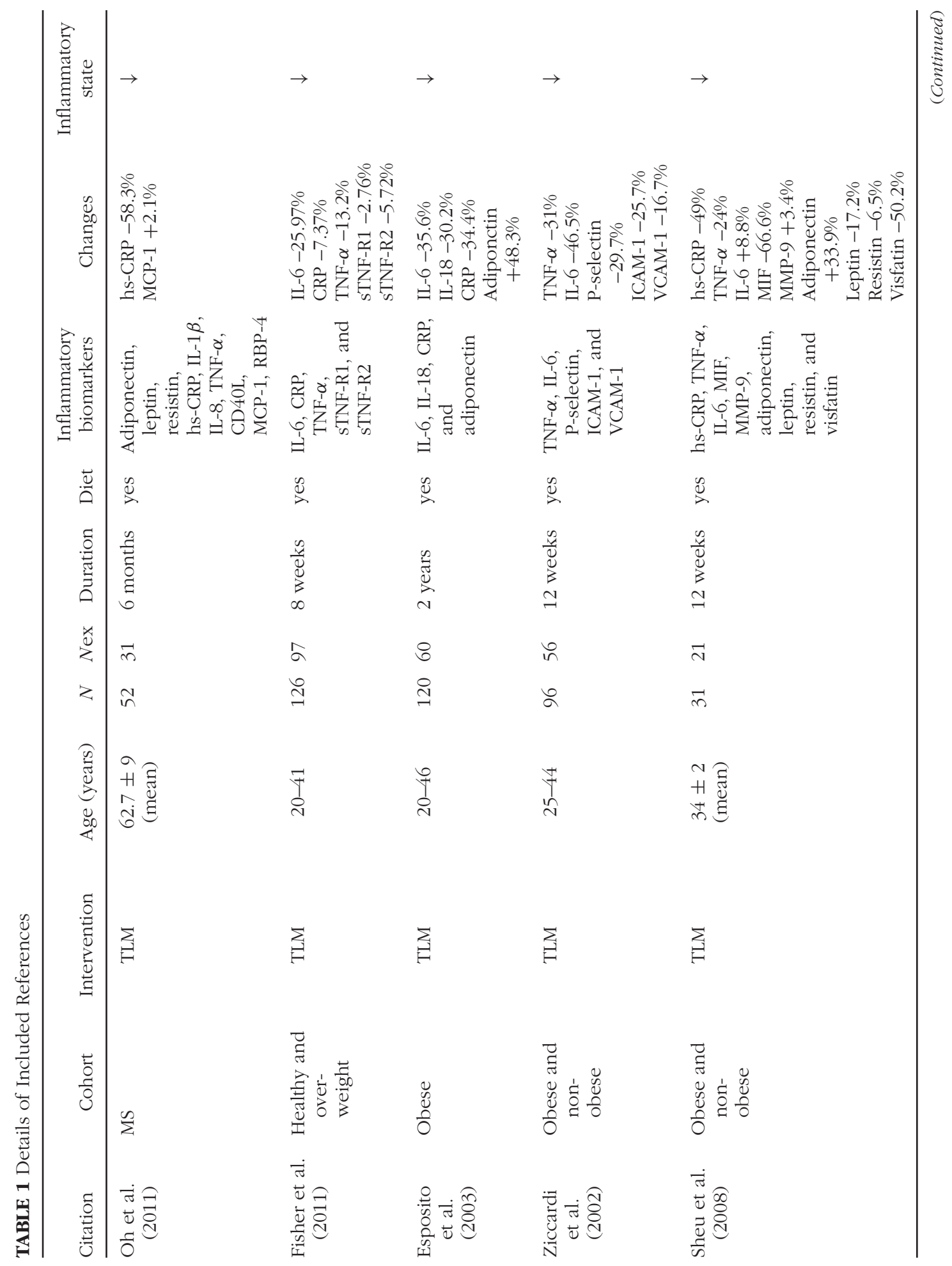




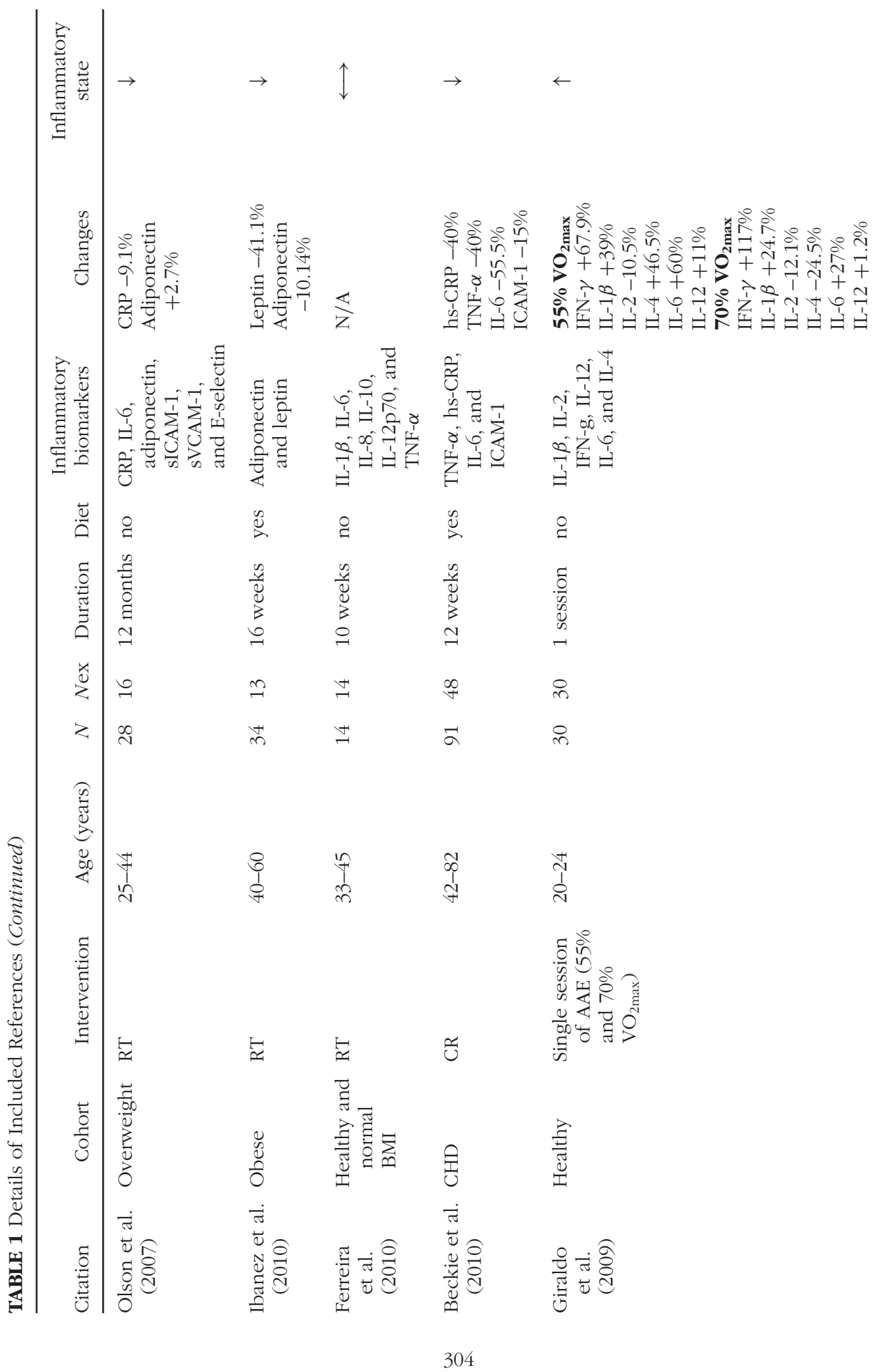




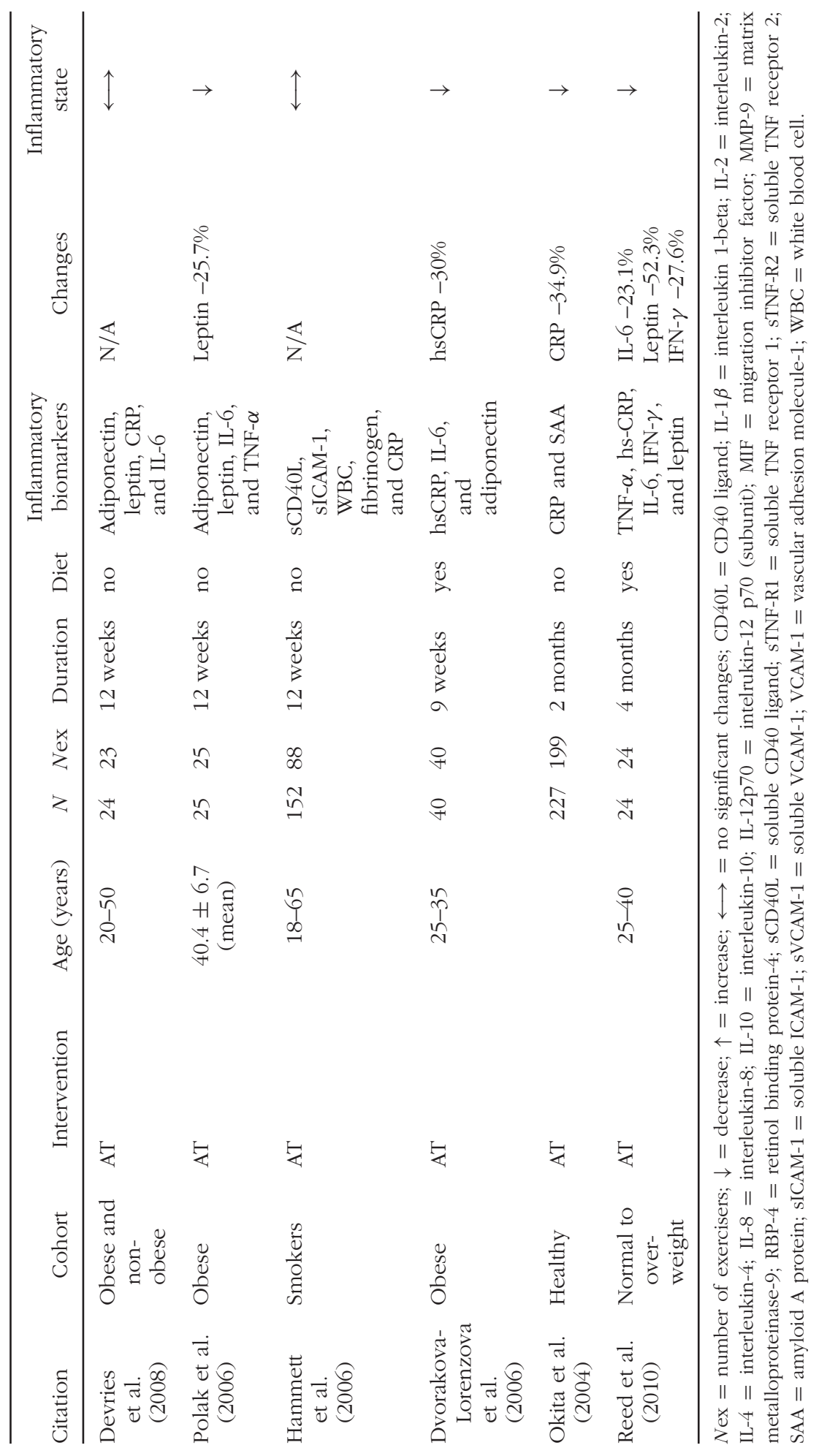


for women with $\mathrm{CHD}$, such as blood pressure and total cholesterol/high density lipoprotein ratio. They supported the benefits of moderate-intensity exercise for reducing inflammation and central obesity in women with established CHD. This study was similar to those involving TLM, using both diet and lifestyle modification strategies.

\section{TLM}

Therapeutic lifestyle intervention is multidisciplinary and should be supported by evidence-based practice guidelines. Its delivery should be based on behavioral readiness to change. Lifestyle programs usually focus on exercise, diet, stress management, and smoking cessation. For this review, TLM studies comprised at least two of the following lifestyle management strategies: diet modification, exercise with or without supervision, recreational activities, health education and counseling, and behavioral or psychological education. Five studies evaluated TLM interventions ranging from eight weeks to two years of duration. Even though the included studies did not follow the same lifestyle management protocol, all reported significant reductions in inflammatory markers, primarily related to weight reduction and changes in fat distribution. These studies had a mean decrease of $30.5 \%$ in inflammatory markers after intervention compared with maintenance of usual habits or given general information (Table 2). Hs-CRP, Il-6, CRP, and TNF- $\alpha$ decreased following TLM by means of $53.7 \%, 24.8 \%, 20.9 \%$, and $22.7 \%$, respectively. However, adiponectin increased by $41.2 \%$ following TLM. Oh et al. (2011) investigated the impact of a six-month therapeutic lifestyle modification program on inflammatory biomarkers in women with MS. The intervention included health screening, exercise, low-calorie diet, health education, and counseling for the study period and instructed the control group to maintain their usual lifestyle behaviors. The exercise protocol used a standard recorded video for all participants, including yoga stretching, rhythmic aerobic dance (Tae-Bo), and 40-minute warm-up and cool-down exercises three times per week in supervised sessions. In addition to the group sessions, daily home-based brisk walking was encouraged. Hs-CRP significantly decreased (58.3\%), and monocyte chemotactic protein-1 (MCP-1) increased by 22.1\%. Both changes were associated with the exercise protocol (Oh et al., 2011). However, most control and intervention group participants were farmers engaged in strenuous physical labor, representing a strong selection bias in study participants that could have provided non-representative study outcomes (Booth \& Roberts, 2008).

Fisher et al. (2011) investigated the effect of diet with or without exercise training on inflammatory markers and fat distribution in healthy overweight women. Participants were randomized to diet only, diet and AT, or diet and RT. Weight reduction was associated with a statistically significant decrease in TNF- $\alpha$ (13.2\%), soluble TNF receptor type 1 (2.76\%), soluble TNF receptor 
TABLE 2 Percent Change in Inflammatory Biomarkers by Intervention Protocol

\begin{tabular}{|c|c|c|c|c|}
\hline Type of intervention & $\begin{array}{l}\text { Inflammatory } \\
\text { biomarker }\end{array}$ & $\begin{array}{l}\text { Percent } \\
\text { change }\end{array}$ & $\begin{array}{l}\text { Biomarker } \\
\text { mean }\end{array}$ & $\begin{array}{l}\text { Protocol } \\
\text { mean }\end{array}$ \\
\hline \multirow[t]{5}{*}{$\begin{array}{l}\text { TLM (Oh et al., } \\
\text { 2011; Esposito } \\
\text { et al., 2003; Fisher } \\
\text { et al., 2011; Sheu } \\
\text { et al., 2008; } \\
\text { Ziccardi et al., } \\
\text { 2002) }\end{array}$} & hs-CRP & $\begin{array}{l}-58.3 \% \\
-49.0 \%\end{array}$ & $-53.7 \%$ & $-30.5 \%$ \\
\hline & IL-6 & $\begin{array}{r}-26.0 \% \\
-35.6 \% \\
-46.5 \% \\
8.8 \%\end{array}$ & $-24.8 \%$ & \\
\hline & CRP & $\begin{array}{r}-7.4 \% \\
-34.4 \%\end{array}$ & $-20.9 \%$ & \\
\hline & $\mathrm{TNF}-\alpha$ & $\begin{array}{l}-13.2 \% \\
-31.0 \% \\
-24.0 \%\end{array}$ & $-22.7 \%$ & \\
\hline & Adiponectin* & $\begin{array}{l}48.5 \% \\
33.9 \%\end{array}$ & $41.2 \% *$ & $41.2 \%{ }^{*}$ \\
\hline $\begin{array}{l}\text { RT (Ferreira et al., } \\
\text { 2010; Ibanez } \\
\text { et al., 2010; Olson } \\
\text { et al., 2007) }\end{array}$ & Adiponectin* & $\begin{array}{r}2.7 \% \\
-10.1 \%\end{array}$ & $-3.7 \% *$ & $-3.7 \% *$ \\
\hline \multirow{2}{*}{$\begin{array}{l}\text { CR (Beckie et al., } \\
\text { 2010) }\end{array}$} & hs-CRP & $-40.0 \%$ & $-40.0 \%$ & $-45.2 \%$ \\
\hline & $\begin{array}{l}\text { TNF- } \alpha \\
\text { IL-6 }\end{array}$ & $\begin{array}{l}-40.0 \% \\
-55.5 \%\end{array}$ & $\begin{array}{l}-40.0 \% \\
-55.5 \%\end{array}$ & \\
\hline \multirow[t]{2}{*}{$\begin{array}{l}\text { AAE (Giraldo et al., } \\
\text { 2009) }\end{array}$} & $\mathrm{IFN}-\gamma$ & $\begin{array}{r}67.9 \% \\
117.0 \%\end{array}$ & $92.5 \%$ & $68.0 \%$ \\
\hline & IL-6 & $\begin{array}{l}60.0 \% \\
27.0 \%\end{array}$ & $43.5 \%$ & \\
\hline $\begin{array}{l}\text { AT(Devries et al., } \\
\text { 2008; Dvorakova- } \\
\text { Lorenzova et al., } \\
\text { 2006; Hammett } \\
\text { et al., 2006; Okita } \\
\text { et al., 2004; Polak } \\
\text { et al., 2006; Reed } \\
\text { et al., 2010) }\end{array}$ & $\begin{array}{l}\text { Leptin } \\
\text { hs-CRP CRP } \\
\text { Il-6 IFN- } \gamma\end{array}$ & $\begin{array}{c}-25.0 \% \\
-52.3 \% \\
-30.0 \% \\
-34.9 \% \\
-23 \% \\
-27.60 \%\end{array}$ & $\begin{array}{l}-38.7 \% \\
-30.0 \% \\
-34.9 \% \\
-23.0 \% \\
-27.6 \%\end{array}$ & $-30.8 \%$ \\
\hline
\end{tabular}

*Anti-inflammatory biomarker.

type 2 (5.72\%), CRP (7.37\%), and Il-6 (25.97\%), regardless of the presence or type of exercise protocol (Fisher et al., 2011). Esposito et al. (2003) investigated the effect of a lifestyle change program designed to obtain and sustain a reduction in body weight on inflammatory biomarkers of obese women. The intervention group received detailed advice about achieving at least a 10\% weight reduction through a low-energy, Mediterranean-style diet and 
physical activity consisting of walking, swimming, and ball games in monthly sessions with a nutritionist and exercise trainer. The control group received general information about healthy diet and exercise. They reported decreases in Il-6 (35.6\%), interleukin 18 (Il-18; 30.2\%), and CRP (34.4\%), significantly decreasing vascular inflammation and cardiovascular risks associated with reducing blood pressure, insulin sensitivity, and lipid and glucose intake in obese women. They also reported that a more pronounced reduction in measured markers occurred in those participants with higher initial levels of the inflammatory markers. Only eight dropouts occurred among 120 participants $(<7 \%)$ after two years of follow-up, three in the intervention group and five in the control group. This attrition rate was much lower than average rates usually found in long-term programs (Dalle Grave et al., 2005). Even the dropouts from the intervention group showed significant decreases in body weight after 24 weeks of follow-up, suggesting acceptability of the intervention and adherence to the lifestyle changes. The authors suggested that weight reduction could be achieved long-term by a multidisciplinary approach and that long duration interventions should be multifactorial to ensure enthusiasm and compliance along the program (Esposito et al., 2003).

Sheu et al. (2008) investigated the association between weight reduction through a low-calorie diet and physical activity in obese versus lean participants. The dietary program consisted of a daily reduction of $500 \mathrm{kcal}-$ $1,000 \mathrm{kcal}$ and increased, although not restricted, physical activity, including regular walking and occasional, relatively non-intensive recreational activities. A 5\% reduction of initial weight in obese women was associated with significant improvements in several pro-inflammatory gene transcriptions (Sheu et al., 2008). Ziccardi et al. (2002) investigated the association between weight reduction and inflammatory cytokine concentrations in obese women and compared their baseline results with those of lean controls. TNF- $\alpha$ and IL-6 were elevated in obese women compared with the lean group and correlated with indexes of adiposity, mainly central obesity. After one year of a weight reduction program, including diet, exercise, and behavioral counseling, only the obese group were reexamined, and all obese participants lost at least $10 \%$ of their original weight, which was associated with a significant down-regulation of their inflammatory status. In addition, a more pronounced decrease in TNF- $\alpha$ and Il-6 was observed in those participants with higher baseline values of these markers (Ziccardi et al., 2002).

\section{AT}

Six studies involved AT, and two of them reported no significant changes in inflammatory states of participants. Okita et al. (2004) investigated whether aerobic exercise training and weight loss could decrease CRP levels in healthy participants. AT consisted of a twice-weekly, supervised, 80-minute aerobic dance workout immediately followed by an additional 
30 to 60 minutes of bicycle or treadmill exercise at $60 \%$ to $80 \%$ of peak heart rate. In addition, recommendations were made to participants to perform home-based exercise at least one day per week for two months. No diet was administered nor was dietary change encouraged. After the training program, all conventional cardiovascular risk variables improved, and CRP levels declined significantly compared with baseline values. However, these changes were not associated with the amount of weight reduction (Okita et al., 2004).

A study by Polak et al. (2006) investigated the effect of AT on plasma levels and subcutaneous abdominal adipose tissue gene expression of inflammatory biomarkers in obese women before and after the intervention. Twenty-five participants underwent a 12 -week aerobic exercise program, five days per week at intensity corresponding to $50 \%$ of individual maximal oxygen consumption, consisting of two days of supervised and three days of unsupervised bicycle ergometer sessions. The study did not include a dietary intervention. After the program, only leptin plasma levels decreased. The authors suggested that the lack of significant changes in other cytokines was due to the type of exercise and duration of the training program as well as sex and degree of obesity of the participants (Polak et al., 2006). However, the small number of participants may have provided insufficient statistical power to detect significant changes in some markers and must be taken into account in interpreting the data.

Hammett et al. (2006) investigated the association between AT for 12 weeks and inflammatory biomarkers associated with cardiovascular risk in female smokers. The exercise group took part in 45-minute supervised sessions three times per week and were free to choose among treadmill, cycle, and rowing ergometers. Participants were encouraged to maintain intensity between $60 \%$ and $70 \%$ of their maximal heart rate. Controls in a health education group were asked to participate in three 45-minute sessions involving group support and general lifestyle advice. Despite a significant improvement in fitness, AT was not associated with decreased inflammatory biomarker levels at either 6 or 12 weeks compared with the control group. Smoking was associated with increases in inflammatory biomarker levels and outweighed the decreased levels associated with exercise training. Further, the duration or intensity of exercise training was insufficient to demonstrate significant changes in inflammatory markers. The association between greater physical fitness and lower inflammatory biomarkers may have been due to long-term regular exercise, which reduced total body and visceral fat (Hammett et al., 2006).

Dvoráková-Lorenzová et al. (2006) investigated circulating CRP levels before and after weight reduction in obese women. The intervention consisted of dietary education combined with increased physical activity. Aerobic exercise training was supervised for one hour, three times per week at a fitness center, while three more sessions of cycling, jogging, or brisk 
walking were unsupervised. Participants were supervised (and advised) to sustain a heart rate of 130 to 135 beats per minute for 45 minutes within 60 minutes of exercise for 9 weeks. They observed a much more pronounced decrease in plasma hs-CRP concentrations in participants with higher initial CRP values but no significant changes in Il-6 concentrations. The reduction in CRP strongly correlated with reduced weight and body adiposity. Also, decreased hs-CRP was associated with changes in plasma triacylglycerols and free fatty acids, so that subclinical inflammation may be an integral part of MS (Dvorakova-Lorenzova et al., 2006).

Devries et al. (2008) investigated the associations of obesity and AT with inflammatory markers in obese and lean females. The training protocol for both groups consisted of 12-week progressive aerobic exercise training commencing with two 15-minute cycling sessions at 50\% $\mathrm{VO}_{2 \text { peak }}$ per week, increasing to three 60-minute sessions at $65 \% \mathrm{VO}_{2 \text { peak }}$. Obese participants showed significantly higher levels of inflammatory biomarkers than lean participants at baseline and after the intervention. However, AT without weight loss had no effect on inflammation. On the other hand, AT lowered systemic oxidative stress in both lean and obese women and showed a significant reduction of progression of obesity to obesity-related metabolic disorders (Devries et al., 2008).

Reed, De Souza, and Williams (2010) hypothesized that exercise combined with calorie restriction would produce favorable changes in inflammatory biomarkers in non-obese sedentary women after the intervention. The exercise protocol consisted of supervised sessions four times per week involving a five-minute warm-up, 40-90 minutes of aerobic activity at $79 \%( \pm 0.7 \%)$ of maximal heart rate, and a 10-minute cool-down. The duration of the exercise sessions was equal to that required to expend a target amount of calories (20\% of baseline energy needs). Significant reductions in Il-6, leptin, and INF- $\gamma$, and weight loss were observed in response to moderate to vigorous AT and calorie restriction, but no association was observed between Il-6 and reduced adiponectin expression (Reed et al., 2010).

\section{RT}

Olson et al. (2007) examined biomarkers of inflammation in healthy overweight women before and after one year of moderate-intensity RT. The RT program consisted of at least two training sessions per week with at least 48 hours between sessions. Each session began with warm-up on a treadmill, cycle ergometer, elliptical trainer, stepper, or a five-minute walk on a track, following deep abdominal and lower back exercises for core stability and injury prevention. Participants performed three sets of 8-10 repetitions using free weights and isotonic variable resistance machines for the major muscle groups. The first 16 weeks of training were supervised. Thereafter, participants completed the RT protocol on their own 
while meeting twice every 12 weeks with the fitness trainer. The control participants received brochures on walking and exercise from the American Heart Association without further instructions. Moderate-intensity RT resulted in modest but significant improvements in inflammatory biomarkers without affecting cell adhesion molecules, supporting the rationale for including RT protocols to help reduce the inflammatory contribution to atherothrombosis in overweight women (Olson et al., 2007).

Ibanez et al. (2010) investigated the association between a combination of 16 weeks of progressive RT and a weight-loss diet and circulating adiponectin in middle-aged obese women. Participants were randomly assigned to one of three groups: control group, diet only, or diet and RT, with the same calorie restriction as the diet alone group and a 16-week supervised, whole-body RT program consisting of two sessions per week. Data collected before and after the intervention showed that weight reduction through RT and diet was related to a reduction in different cardiovascular risk factors (insulin sensitivity, visceral fat, and low-density lipoprotein cholesterol, among others) in spite of a significant decrease in adiponectin levels (Ibanez et al., 2010).

Ferreira et al. (2010) evaluated whether a 10-week circuit RT protocol would decrease body mass and percent body fat in sedentary and healthy women without inducing inflammatory responses. The circuit included nine exercise stations, and participants performed one round of 15 to 20 warmup repetitions and two rounds of 8 to 12 repetition maxima three times per week. Loads were adjusted to obtain maximum effort and appropriate execution. Data collected before and after the intervention showed a decrease in percent body fat and an increase in lean body mass without changing total body mass. No significant modifications were reported in inflammatory biomarkers. The authors suggested this was due to the structure of the program represented by a proper exercise sequence (alternating muscle group agonists) and training frequency (with a 48-hour recovery between sessions), providing participants with sufficient physical recovery time and adapting them to the minimum inflammation response (Ferreira et al., 2010).

\section{AAE}

Only one study analyzed the effect of AAE on inflammatory biomarkers. Giraldo et al. (2009) evaluated differences in the magnitude of exerciseinduced stimulation of the innate and/or inflammatory response following moderate and intense exercise. Healthy sedentary women were randomly assigned to one of two groups, 55\% $\mathrm{VO}_{2 \max }$ and $70 \% \mathrm{VO}_{2 \max }$. A single bout of moderate exercise induced a statistically significant better innate/inflammatory response than an intense bout, resulting in a better profile of the systemic release of pro- and anti-inflammatory cytokines (Giraldo et al., 2009). 


\section{DISCUSSION}

The purpose of this review was to perform a systematic critical review of the literature on the effect of different types of acute and chronic physical activity on inflammatory biomarkers in females. The main findings were that (a) integrative approaches, such as TLM or CR, resulted in major decreases in inflammatory biomarkers; (b) AAE was associated with significantly increased levels of inflammatory biomarkers; (c) most RT protocols reported no significant changes; and (d) AT protocols reported important decreases in inflammatory biomarkers coupled with weight reduction. These observations suggest that integrative interventions, including diet and moderate exercise, with particular attention to AT, appeared to be the most effective in improving inflammatory biomarkers in women with or without metabolic disorders.

According to Peter D. Gluckman and Mark A. Hanson (2004), epidemiologic observations led to the hypothesis that the risk of developing metabolic disorders in adulthood is related not only to genetic and adult lifestyle factors but also to environmental factors (Gluckman \& Hanson, 2004). Sedentary lifestyle can significantly increase circulating inflammatory biomarkers. Evidence garnered from epidemiologic prospective cohort and intervention studies suggested that chronic diseases were associated with physical inactivity (Hilberg, 2008; Petersen \& Pedersen, 2005; Booth et al., 2002; Booth \& Roberts, 2008). Inflammation is also modulated by diet, thus changes in diet may be associated with changes in inflammatory markers (Cavicchia et al., 2009).

\section{CONCLUSIONS}

Most findings showed that most of the relation of exercise training or lifestyle modification to inflammatory biomarkers was observed in women with higher initial values of inflammatory biomarkers. The integrative approaches, such as TLM or CR, showed major changes in inflammatory biomarkers as long as moderate-intensity exercise was involved. Secondary lifestyle characteristics, such as hard physical labor or smoking, were associated with systemic inflammation. While regular strenuous physical labor improved, smoking habits diminished the beneficial effects of exercise on inflammatory markers. Most studies reported positive changes in inflammatory biomarkers associated with weight reduction (5\% to 10\%), regardless of exercise. Circuit RT can improve body composition without increasing inflammatory biomarkers if the proper combination of exercise sequence and adequate rest is met. Moderate exercise appears to be the best choice even in acute exercise bouts.

These observations suggest that integrative interventions, including diet, moderate exercise (with a major focus on AT between $60 \%$ to $80 \%$ of 
maximum heart rate or $50 \%$ to $60 \%$ of $\mathrm{VO}_{2 \max }$ ) and circuit $\mathrm{RT}$ (with 8 to 10 exercises, 8 to 12 repetitions of alternating segments with at least 48 hours of rest), health education, and counseling are needed to achieve at least 5\% to $10 \%$ body weight reduction. These interventions appear to be the most effective strategies to improve inflammatory outcomes in women.

\section{LIMITATIONS AND FUTURE DIRECTIONS}

Some limitations of this critical review should be noted. First, dietary effects were monitored in only a few studies using exercise as the sole intervention, thereby potentially introducing threats to the validity of the studies reviewed here by confounding the true relationship with exercise. More studies with controlled diets are needed to clarify the association between exercise protocols alone and inflammatory biomarkers. Second, few studies evaluated the distinction between alterations in visceral and subcutaneous fat versus changes in body weight. Future studies using image analysis techniques also must be undertaken to elucidate the effect of body composition changes on cytokine levels. Finding the ideal combination of type, duration, and intensity of exercise protocols and diet may also potentially contribute to better health outcomes by improving inflammatory states. Moreover, none of the studies reviewed considered the influence of menstrual phases in which dietary and/or physical activity interventions occurred, and such influences must be taken into account for female populations. Additional methodological limitations of some of the studies included the failure to use masked outcome assessors in randomized trials or to report compliance. Betweengroup statistical analysis similarly was not routinely performed in all studies reviewed. Furthermore, inadequately described training volumes and intensities along with a number of confounders, such as protocols employing diet and exercise together, as well as not fully examining the potential effect modification by smoking and other lifestyle and work habits, compromised the quality of some trials. The lack of acute resistance exercise to clarify the effect of this type of intervention on inflammatory biomarkers also must be addressed.

\section{REFERENCES}

Aldhahi, W., and O. Hamdy. 2003. Adipokines, inflammation, and the endothelium in diabetes. Curr Diab Rep 3(4):293-8.

Beckie, T. M., J. W. Beckstead, and M. W. Groer. 2010. The influence of cardiac rehabilitation on inflammation and metabolic syndrome in women with coronary heart disease. J Cardiovasc Nurs 25(1):52-60. 
Bengmark, S. 2006. Impact of nutrition on ageing and disease. Curr Opin Clin Nutr Metab Care 9(1):2-7.

Booth, F. W., M. V. Chakravarthy, and E. E. Spangenburg. 2002. Exercise and gene expression: Physiological regulation of the human genome through physical activity. J Physiol 543(Pt 2):399-411.

Booth, F., and C. K. Roberts. 2008. Linking performance and chronic disease risk: Indices of physical performance are surrogates for health. BrJ Sports Med 42:950-2.

Cavicchia, P. P., S. E. Steck, T. G. Hurley, J. R. Hussey, Y. Ma, I. S. Ockene, and J. R. Hebert. 2009. A new dietary inflammatory index predicts interval changes in serum high-sensitivity C-reactive protein. J Nutr 139(12):2365-72.

Dalle Grave, R., S. Calugi, E. Molinari, M. L. Petroni, M. Bondi, A. Compare, and G. Marchesini. 2005. Weight loss expectations in obese patients and treatment attrition: An observational multicenter study. Obes Res 13(11):1961-9.

De Morton, N. A. 2009. The PEDro scale is a valid measure of the methodological quality of clinical trials: A demographic study. Aust J Physiother 55(2):129-33.

Devries, M. C., M. J. Hamadeh, A. W. Glover, S. Raha, I. A. Samjoo, and M. A. Tarnopolsky. 2008. Endurance training without weight loss lowers systemic, but not muscle, oxidative stress with no effect on inflammation in lean and obese women. Free Radic Biol Med 45(4):503-11.

Dvorakova-Lorenzova, A., P. Suchanek, P. J. Havel, P. Stavek, L. Karasova, Z. Valenta, et al. 2006. The decrease in C-reactive protein concentration after diet and physical activity induced weight reduction is associated with changes in plasma lipids, but not interleukin-6 or adiponectin. Metabolism 55(3):359-65.

Esposito, K., A. Pontillo, C. Di Palo, G. Giugliano, M. Masella, R. Marfella, and D. Giugliano. 2003. Effect of weight loss and lifestyle changes on vascular inflammatory markers in obese women: A randomized trial. JAMA (14). Retrieved from http://www.mrw.interscience.wiley.com/cochrane/clcentral/articles/482/ CN-00436482/frame.html.

Ferreira, F. C., A. I. de Medeiros, C. Nicioli, J. E. Nunes, G. E. Shiguemoto, J. Prestes, et al. 2010. Circuit resistance training in sedentary women: Body composition and serum cytokine levels. Appl Physiol Nutr Metab 35(2):163-71.

Fisher, G., T. C. Hyatt, G. R. Hunter, R. A. Oster, R. A. Desmond, and B. A. Gower. 2011. Effect of diet with and without exercise training on markers of inflammation and fat distribution in overweight women. Obesity 19(6):1131-6.

Garruti, G., S. Cotecchia, F. Giampetruzzi, F. Giorgino, and R. Giorgino. 2008. Neuroendocrine deregulation of food intake, adipose tissue and the gastrointestinal system in obesity and metabolic syndrome. J Gastrointestin Liver Dis 17(2):193-8.

Giraldo, E., J. J. Garcia, M. D. Hinchado, and E. Ortega. 2009. Exercise intensitydependent changes in the inflammatory response in sedentary women: Role of neuroendocrine parameters in the neutrophil phagocytic process and the pro-/anti-inflammatory cytokine balance. Neuroimmunomodulation 16(4):237-44.

Gluckman, P. D., and M. A. Hanson. 2004. Living with the past: Evolution, development, and patterns of disease. Science 305(5691):1733-6. 
Hammett, C. J. K., H. Prapavessis, J. C. Baldi, N. Varo, U. Schoenbeck, R. Ameratunga, et al. 2006. Effects of exercise training on 5 inflammatory markers associated with cardiovascular risk. Am Heart J 151(2):367.e7-367.e16.

Hilberg, T. 2008. Physical activity in the prevention of cardiovascular diseases. Epidemiology and mechanisms. Hamostaseologie 28(1-2):9-12, 14-5.

Ibanez, J., M. Izquierdo, C. Martinez-Labari, F. Ortega, A. Grijalba, L. Forga, et al. 2010. Resistance training improves cardiovascular risk factors in obese women despite a significative decrease in serum adiponectin levels. Obesity 18(3):535-41.

Lacasa, D., S. Taleb, M. Keophiphath, A. Miranville, and K. Clement. 2007. Macrophage-Secreted factors impair human adipogenesis: Involvement of proinflammatory state in preadipocytes. Endocrinology 148(2):868-77.

Lazar, M. A. 2005. How obesity causes diabetes: Not a tall tale. Science 307(5708):373-5.

Oh, E. G., S. Y. Bang, S. H. Kim, S. S. Hyun, S. H. Chu, Y. K. Jeon, et al. 2011. Therapeutic lifestyle modification program reduces plasma levels of the chemokines CRP and MCP-1 in subjects with metabolic syndrome. Biol Res Nurs. Retrieved from http://www.ncbi.nlm.nih.gov/entrez/ query.fcgi? $\mathrm{cmd}=$ Retrieve $\& \mathrm{db}=$ PubMed\&dopt=Citation\&list_uids $=21859748$.

Okita, K., H. Nishijima, T. Murakami, T. Nagai, N. Morita, K. Yonezawa, et al. 2004. Can exercise training with weight loss lower serum C-reactive protein levels? Arterioscler Thromb Vasc Biol 24(10):1868-73.

Olson, T. P., D. R. Dengel, A. S. Leon, and K. H. Schmitz. 2007. Changes in inflammatory biomarkers following one-year of moderate resistance training in overweight women. Int J Obes (Lond) 31(6):996-1003.

Petersen, A. M., and B. K. Pedersen. 2005. The anti-inflammatory effect of exercise. J Appl Physiol 98(4):1154-62.

Polak, J., E. Klimcakova, C. Moro, N. Viguerie, M. Berlan, J. Hejnova, et al. 2006. Effect of aerobic training on plasma levels and subcutaneous abdominal adipose tissue gene expression of adiponectin, leptin, interleukin 6, and tumor necrosis factor alpha in obese women. Metabolism 55(10):1375-81.

Reed, J. L., M. J. De Souza, and N. I. Williams. 2010. Effects of exercise combined with caloric restriction on inflammatory cytokines. Appl Physiol Nutr Metab 35(5):573-82.

Schulz, K. F., D. G. Altman, D. Moher, and Consort Group. 2010. CONSORT 2010 statement: Updated guidelines for reporting parallel group randomised trials. PLoS Med 7(3):e1000251.

Sheu, W. H., T. M. Chang, W. J. Lee, H. C. Ou, C. M. Wu, L. N. Tseng, et al. 2008. Effect of weight loss on proinflammatory state of mononuclear cells in obese women. Obesity 16(5):1033-8.

Silva, T. S., C. A. Longui, C. D. Faria, M. N. Rocha, M. R. Melo, T. G. Faria, et al. 2008. Impact of prolonged physical training on the pituitary glucocorticoid sensitivity determined by very low dose intravenous dexamethasone suppression test. Horm Metab Res 40(10):718-21.

Skilton, M. R., D. P. Sieveking, J. A. Harmer, J. Franklin, G. Loughnan, S. Nakhla, et al. 2008. The effects of obesity and non-pharmacological weight loss on vascular and ventricular function and structure. Diabetes Obes Metab 10(10):874-84. 
Smith, D. T., L. J. Carr, C. Dorozynski, and C. Gomashe. 2008. Internet-delivered lifestyle physical activity intervention: Limited inflammation and antioxidant capacity efficacy in overweight adults. J Appl Physiol 106(1):49-56.

Troseid, M., K. T. Lappegard, T. E. Mollnes, H. Arnesen, and I. Seljeflot. 2009. The effect of exercise on serum levels of interleukin-18 and components of the metabolic syndrome. Metab Syndr Relat Disord 7(6):579-84.

Verhagen, A. P., H. C. de Vet, R. A. de Bie, A. G. Kessels, M. Boers, L. M. Bouter, and P. G. Knipschild. 1998. The Delphi list: A criteria list for quality assessment of randomized clinical trials for conducting systematic reviews developed by Delphi consensus. J Clin Epidemiol. Retrieved from http://www.ncbi.nlm.nih.gov/entrez/query.fcgi? $\mathrm{cmd}=$ Retrieve\& $\mathrm{db}=$ PubMed\&dopt $=$ Citation\&list_uids $=10086815$.

Ziccardi, P., F. Nappo, G. Giugliano, K. Esposito, R. Marfella, M. Cioffi, et al. 2002. Reduction of inflammatory cytokine concentrations and improvement of endothelial functions in obese women after weight loss over one year. Circulation (7). Retrieved from http://www.mrw.interscience.wiley.com/ cochrane/clcentral/articles/087/CN-00378087/frame.html. 